$\Rightarrow$ DEVELOPMENT

\title{
Modelling disease in kidney organoids
}

Recent studies have described the generation of kidney organoids from human pluripotent stem cells (hPSCs) and the successful development of nephron segments within these organoids. Among these, a new study has described the use of gene editing to model genetic kidney disease in 3D kidney organoids generated from hPSCs. "Our most exciting finding was that the CRISPR-mutant

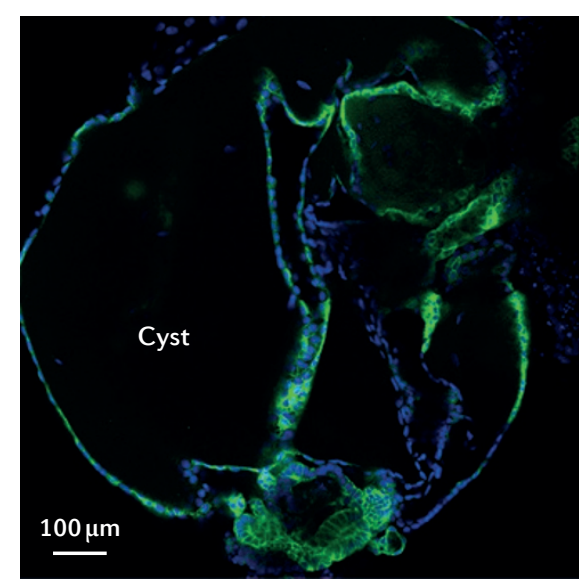

A cyst associated with PKD1 deletion. Permission obtained from Nature Publishing Group (C) Freedman, B. S. et al. Nat. Commun. 6, 8715 (2015). organoids can recapitulate features of genetic kidney disease," says researcher Benjamin Freedman. "These findings teach us that principal features of kidney architecture and disease are cell-intrinsic."

Freedman and colleagues devised a 3D culture system to promote the maturation of hPSCs into kidney organoids, to mimic the 3D architecture of the nephron. "We have been excited about hPSCs for several years now because they represent a renewable source of patient-matched tissues and present an opportunity to create human kidney tissue in the lab, which we can then use to study the root causes of disease," explains Freedman. To generate the 3D kidney structures, the researchers used a two-step procedure involving the formation of epiblast spheroids from hPSCs followed by GSK $\beta$ inhibition to initiate the development of tubular organoids. "We were very impressed by the ability of these mini-kidney organoids to recreate 3D features of kidney architecture," says Freedman. "The organoids formed long, segmented tubules, which resembled nephrons both in patterning and in their response to nephrotoxic chemicals."
To model genetic kidney disease in hPSCs, the team adopted a genome editing approach using CRISPR-Cas9 technology to introduce targeted, loss-of-function mutations into genes linked to either polycystic kidney disease (PKD) or focal segmental glomerulosclerosis (FSGS). "In organoids with PKD-inducing mutations, we observed cyst formation from tubular epithelial cells whereas in organoids with FSGS-related mutations, we observed junctional defects in podocytes," says Freedman. "These phenotypes are very similar to what we might expect to see in knockout mice or in patients with these diseases; importantly, disease phenotypes were not observed in nonmutant controls."

The researchers hope that further studies will provide novel insights into cellular pathways that might be amenable to therapeutic intervention. "The system is potentially high-throughput, and an exciting possibility is to screen for new drugs capable of rescuing injury and disease phenotypes," says Freedman. "We are also interested in using CRISPR as a gene therapy tool, to correct genetic mutations."

Susan J. Allison

ORIGINAL ARTICLE Freedman, B. S. et al. Modelling kidney disease with CRISPR-mutant kidney organoids derived from human pluripotent epiblast spheroids. Nat. Commun. 6, 8715 (2015) 